\title{
Consent for clinical research in the neonatal intensive care unit: a retrospective survey and a prospective study
}

\author{
E Burgess, N Singhal, H Amin, D D McMillan, H Devrome
}

Arch Dis Child Fetal Neonatal Ed 2003;88:F280-F286

Background: Recruitment into research studies in the neonatal intensive care unit has been problematic. Therefore suggestions have been made to take decision making about enrolment out of the hands of the parents.

Objective: To understand parental perceptions of the process of recruitment and enrolment for research in the neonatal intensive care unit.

Method: A questionnaire was developed and used in both a retrospective survey and a prospective study of parents whose newborns were enrolled in trials in a neonatal intensive care unit. Closed ended and open ended questions were included, as well as demographic questions.

Results: The retrospective survey had a $79 \%$ response rate (29 of 38 ). Overall, $90 \%$ of parents felt that they had made informed decisions, and $93 \%$ were against the option that a doctor decide if the newborn should be enrolled into a study, rather than the parent. Although some parents $(38 \%)$ found that recruitment did add "stress to an already stressful situation", $90 \%$ felt that they had made informed decisions and understood the elements of the study. Most parents had been requested to enrol their newborn into more than one trial, and, on average, they thought that they would be comfortable with enrolment into two studies (range 0-6). When asked how the process could be improved, parents suggested that information be made available before delivery. The responses of parents in the prospective study were mostly consistent with those from the retrospective survey.

See end of article for authors' affiliations

Correspondence to:

DrE Burgess, 140329

Street NW, Calgary, $A B$,

Canada T2N 2T9:

ellen.burgess@

CalgaryHealthRegion.ca

Conclusions: Overall the parents did not support the suggestion that decision making about enrolment be taken away from parents and put into the hands of doctors. The healthcare team should support

Accepted

25 October 2002 parents in their role of decision maker, enhance availability of the research staff, and provide more information about the research.

T he opportunities for advancements in the prevention and treatment of diseases that affect children or begin in childhood have never been greater. ${ }^{1}$ Advances in clinical medicine are often the result of randomised clinical trials. ${ }^{2}$ However, studies on the newborn are limited. Enrolment of patients into randomised clinical trials almost always requires informed consent of the patient. In the case of children or newborns, the patient himself/herself is unable to give consent. The parent has traditionally and legally given consent/permission for medical care, and hence the parent is looked to for permission to enrol the child into a research trial. Research in children is permitted only if the child is subjected to no more than minimal risk and stands to benefit from the study. ${ }^{3}$

The informed consent process, considered a crucial element in the protection of research subjects, has three important components: information, capacity or competence, and voluntariness. It has been suggested that parents are so anxious about the welfare of their newborn that they are not consent competent and their consent may not truly be voluntary. Clinical trialists concerned about the low or poor recruitment rates in neonatal intensive care unit (NICU) clinical trials have argued that parents are not sufficiently educated to understand medical issues surrounding the care of their newborn and the need for research, ${ }^{3}$ nor would they be able to understand complex medical information; hence their consent/permission would not be appropriately "informed". ${ }^{4}$ In Australian studies of parents who had, and had not, enrolled their children in an asthma trial, those who had given consent/permission were found to have less formal education than those who had refused. Also, the "consenting" parents were found to have more reliance on the healthcare system. ${ }^{67}$ The need for informed consent was felt to act as a "social fil- ter" biasing recruitment to the less well educated families. ${ }^{8}$ Another hypothesis put forward is that parents with less education may not have access to good health care for their children and therefore would attempt to access health care through participation in research.

Alternatives such as enrolment without consent/ permission, ${ }^{3}$ having an independent group of experts give consent, ${ }^{45}$ and providing increased information about trials for the general public ${ }^{4}$ have been suggested as possible remedies.

The views and perceptions of parents about research benefits and their participation as proxy consent givers have been assessed in only a few studies. ${ }^{6}{ }^{11}{ }^{11}$ The objective of this study was to understand the parents' perception of the consent process, and their level of comfort and autonomy in making the decision to enrol their newborn into a research study.

\section{METHODS}

The NICU at Foothills Hospital is the only tertiary care referral centre for a population base of 1.2 million Canadians living in southern Alberta, with 19000 obstetrical deliveries annually. Both studies were reviewed and approved by the research and development committee of Foothills Hospital, and the conjoint medical research ethics board of Foothills Hospital and the University of Calgary.

\section{Questionnaire development}

Two meetings were held with the parent support group of the NICU at Foothills Hospital in order to understand the parents' concerns and develop the questionnaire. The questionnaire included 35 questions; responses were on a five point Likert 
Table 1 Parental views and understanding of the recruitment process

\begin{tabular}{|c|c|c|c|c|}
\hline \multirow[b]{2}{*}{ Category/question } & \multicolumn{2}{|c|}{ Retrospective } & \multicolumn{2}{|c|}{ Prospective } \\
\hline & No & $\%$ & No & $\%$ \\
\hline \multicolumn{5}{|l|}{ Autonomy } \\
\hline Doctor should make decision & $2 / 29$ & 7 & $4 / 44$ & 9 \\
\hline Felt pressured to enrol & $10 / 29$ & 34 & $13 / 44$ & 30 \\
\hline \multicolumn{5}{|l|}{ Level of comfort } \\
\hline Made an informed decision & $26 / 29$ & 90 & $40 / 44$ & 91 \\
\hline Comfortable with the decision & $26 / 29$ & 90 & $41 / 44$ & 93 \\
\hline Calm at the time of decision & $17 / 29$ & $59 *$ & $41 / 44$ & 93 \\
\hline \multicolumn{5}{|l|}{ Understanding of decision making } \\
\hline Gave consent for research, not clinical care & $27 / 29$ & 93 & $44 / 44$ & 100 \\
\hline Knew purpose & $29 / 29$ & 100 & $40 / 43$ & 93 \\
\hline Knew procedures & $27 / 29$ & 93 & $36 / 42$ & 86 \\
\hline Knew alternatives & $22 / 29$ & 76 & $17 / 32$ & 53 \\
\hline Research has risks & $15 / 29$ & 52 & $28 / 42$ & 67 \\
\hline Knew could withdraw baby & $20 / 29$ & 69 & $38 / 42$ & 90 \\
\hline Baby may benefit directly & $16 / 29$ & 55 & $29 / 44$ & 66 \\
\hline \multicolumn{5}{|l|}{ Doctor-parent communication } \\
\hline Felt comfortable to ask questions & $28 / 29$ & 97 & $43 / 44$ & 98 \\
\hline Questions were answered & $28 / 29$ & 97 & $43 / 44$ & 98 \\
\hline Doctor used language you understood & $24 / 29$ & 83 & $41 / 44$ & 93 \\
\hline
\end{tabular}

scale from "strongly agree" to "strongly disagree". Four health professionals reviewed it for readability, and 12 healthcare providers (doctors, nurses, community health science students) were asked to review it for face validity and to assign the questions to one of five major themes: (a) parental feelings of autonomy; $(b)$ the level of comfort of the parent; $(c)$ understanding around decision making; (d) perception of the recruitment process including the timing of the request for parental consent/permission, the consent form, and feelings of coercion; (e) parent-doctor communication. There was general agreement about the assignment of the questions to the major theme categories.

\section{Study processes}

The parent who had given consent for the newborn to be in the trial was asked to complete the questionnaire. Information gathered included age and parity of the mother, level of education of the parents, and their site of residence (urban or rural), whether they had previously been involved in a research project or were employed in health care. Three open ended questions asked parents if changes should be made to the recruitment and consent process.

\section{Retrospective survey}

As these parents may have enrolled their newborn(s) into one or more trials, the questionnaire asked them to consider their perceptions of the recruitment into the first trial. As more than one trial was being conducted during the period of recruitment, the parents would not necessarily be responding about the same trial. The timing of consent and risk/benefit ratios of the different trials varied. The clinical trials being conducted over the study period included the use of surfactant, modes of ventilation, nitric oxide therapy, and feeding strategies. Questionnaires were mailed to parents of newborns who had been enrolled into trials over the previous year and could be contacted.

\section{Prospective study}

This study was conducted in association with a prospective randomised trial on the administration of arginine to prevent necrotising enterocolitis in which there was a theoretical risk of intracerebral haemorrhage occurring in 3-5 day old newborns if they received arginine. The parents were approached by the doctor/investigator and then given a day to decide whether to enrol their baby into the arginine trial. The parents who agreed, and those who refused, to enrol their newborns were approached to complete a consent study questionnaire.

\section{Analysis}

Responses were collapsed into "agree" or "disagree"; the middle response was "neutral". Descriptive statistics were used to assess responses to the questions. The response patterns between parents in the retrospective and prospective studies were compared using $\chi^{2}$ testing. Factor analysis was conducted on the responses from the retrospective survey to assess the appropriateness of the groupings of the questions around the five themes described above.

\section{RESULTS}

\section{Retrospective study}

Over the one year period before the retrospective survey, 55 babies had been entered into studies in the NICU; addresses were obtained for 43 of them. Questionnaires were not sent to the parents of five babies who had died as it was thought that this could have been upsetting to them. Therefore questionnaires were sent to 38 sets of parents. Thirty of the 38 questionnaires were returned $(79 \%)$, although one had no responses. Results are reported from 29 respondents.

The consent form was signed by the father in $41 \%$ of the cases and by the mother in 52\%; two respondents did not answer. The age of the mother ranged from 21 to 41 years (mean age 30 years). In most cases (55\%), the mother was primiparous; 11 (40\%) were multiparous, and two did not respond. All but two (27 of 29,93\%) parents had a high school diploma, and 17 (59\%) had post-secondary education. Seven $(25 \%)$ had been in a research study previously, and eight $(28 \%)$ noted that someone in their family had been in a research study previously. Only two (7\%) respondents worked in health care. The mean gestational age of the newborns born to the responding parents was 29 weeks, with 26 born prematurely ( 21 were born before 30 weeks gestation).

The responses of the parents to the retrospective survey are grouped by theme (tables 1 and 2 ).

\section{Autonomy}

Most parents (93\%) disagreed with the idea of the doctor making the decision to enrol the newborn into a research 
study and then informing the parents. Twenty of the 29 parents $(69 \%)$ did not feel pressure to enrol their newborn into a research trial.

\section{Level of comfort}

Most parents (90\%) felt that they had made an informed decision, and were comfortable with the decision made. Over half $(59 \%)$ said that they were calm at the time that they made the decision.

\section{Understanding around decision making}

A large majority of parents $(93 \%)$ reported that they understood that they were giving consent for research as opposed to consent for clinical care. All parents agreed that they knew the purpose of the research, and 93\% reported that they knew what procedures were involved in the study, although a smaller number $(76 \%)$ knew what alternatives were available. About half (52\%) understood that there were risks associated with the research. It was felt by $55 \%$ that the baby would benefit directly from being in the study. Even though it was clearly stated in the consent form, only 69\% of parents indicated that they knew they could withdraw the baby from the study.

\section{Perception of the recruitment process}

(a) Timing: almost half (45\%) of the parents felt that they were expected to give an immediate decision, and just $62 \%$ reported that they had adequate time to ask all their questions. Many (76\%) had a chance to discuss enrolment of their newborn into a trial with their companion/spouse.

(b) Consent forms: most parents (86\%) responded that detailed consent forms were necessary, specifically for parents to protect their children. Almost all parents (83\%) reported that they had read the entire consent form before consenting. Only $66 \%$ of parents remembered being given a copy of the consent form for their records, even though giving a copy of the consent form to the parent is routine practice.

(c) Coercion: a majority of parents $(79 \%)$ responded that they knew that they had the right to refuse to enrol their baby in the trial. One third replied that they had enrolled their baby into the study because they thought that the baby would get better care in the study. Less than half of the parents (38\%) felt that being asked about enrolment "added stress to an already stressful situation", and 31\% felt pressured to give consent for their baby to be enrolled into the clinical trial.

\section{Parent-doctor communication}

All but one parent said that they felt comfortable to question the doctor and that their questions were answered. Most parents $(83 \%)$ reported that the doctor used language that they could understand.

\section{Analysis}

Analysis of the questionnaires for assessment of the parents' perception of the recruitment and consent process used five theme based groupings. Factor analysis revealed good correlation: (a) comfort with autonomous decision making (Cronbach $\alpha=0.86)$; $(b)$ coercion $(\alpha=0.86) ;(c)$ understanding around decision making $(\alpha=0.62)$; $(d)$ recall of formalities $(\alpha=0.72)$; (e) parent-doctor communication $(\alpha=0.72)$.

\section{Number of study recruitment requests}

Most parents responded to the questions about being asked to enrol their newborns into additional studies when the baby was already in one study. About half responded that they felt comfortable being asked for the baby to be in another study, although four parents reported that they were not comfortable with such requests. When asked if they felt "more confident asking questions and making decisions about these additional studies", 39\% reported that they did, but 14\% responded that they did not feel more confident. A small number of parents agreed that participation in these additional studies was influenced by how well their baby did, but 36\% disagreed.

Most parents remembered how many studies in which they had been requested to enrol their baby: one third were asked to enrol in three or more, one third were asked to enrol in two, and one third were asked to enrol in one. Overall, 14 parents consented for their newborn to be enrolled into only one study, two parents consented for two studies, and six parents consented to three or more studies. When asked how many studies that they would feel comfortable with, one third of parents responded two studies, although the responses ranged from zero to six (one parent each responded zero and six).

In response to the open ended questions about changes that could be made in the recruitment process, parents suggested that contact before delivery to inform them about the studies that were recruiting in the NICU and potentially appropriate for their newborn could be helpful. Because enrolment may need to be carried out urgently when there are other stresses for the mother (like her medical condition, pain, use of analgesics, sleep deprivation), one parent asked that someone from the research team return the next day to reiterate the information and answer any questions that may have arisen. Two parents suggested that clearer explanations about the background theory and hypothesis be provided, but that medical jargon be avoided. Two other parents wanted reassurance that when the research was designed to compare two active treatments, that the question was which treatment worked better; presumably this was to reassure them that the baby would not be left without some appropriate active treatment.

When asked about why parents may have refused when asked for consent to enrol their baby into additional studies, it appeared that "discomfort" may have been more important than "risk" - for example, "We were asked to participate in a blood sample-taking type of study. We felt that we had done our bit and the girls didn't need any more needles." One parent was concerned that their newborn was being viewed as a "guinea pig"-_.'I didn't like the second study because it made me feel like they were going to use my baby as a guinea pig."

Parents seemed very interested in receiving "results" of the research, or at least of the progress of their child/ren in the research trial, but did appear to appreciate how long it can take to get an answer from a research study. As one parent remarked, "we made the decision to enrol as we understood that previous studies were the cause of the high level of care provided." The parents of twins wondered if twins could be randomised as a set rather than as individual patients. They said "it is a natural reaction for parents to ask why both twins are not getting the same treatment when doctors have obviously theorised that (it) is better." They wrote that their twins were not randomised to the same treatment and one twin remained on ventilation for two extra weeks and developed more long term problems; these parents could not help but question whether this was circumstantial or the result of the treatment.

\section{Prospective study}

Over a one and a half year period, 73 parents had newborns who were eligible to be enrolled into the clinical trial with which our consent study was associated. Three parents (meaning pairs of parents when both mother and a partner were available) did not speak enough English to complete the survey forms; six had their newborns transferred to other hospitals before final decisions were made about enrolment into the trial. The remaining $64(88 \%)$ were approached to complete questionnaires for this study. Forty four parents (67\%) consented to the clinical trial and completed the survey; six parents $(9 \%)$ consented to the clinical trial but would not complete the survey forms. Fourteen parents refused for their 
Table 2 Parental perceptions of the enrolment process

\begin{tabular}{|c|c|c|c|c|}
\hline \multirow[b]{2}{*}{ Category/question } & \multicolumn{2}{|c|}{ Retrospective } & \multicolumn{2}{|c|}{ Prospective } \\
\hline & No & $\%$ & No & $\%$ \\
\hline \multicolumn{5}{|l|}{ Timing } \\
\hline Had time to talk to partner & $22 / 29$ & 76 & $39 / 42$ & 93 \\
\hline Felt they had to make immediate decision & $13 / 29$ & $45^{*}$ & $4 / 44$ & 9 \\
\hline Adequate time for questions & $18 / 29$ & 62 ** & $38 / 44$ & 86 \\
\hline \multicolumn{5}{|l|}{ Coercion } \\
\hline Knew had right to refuse & $23 / 29$ & 79 & $41 / 43$ & 95 \\
\hline Get better care in study & $10 / 29$ & 34 & $17 / 40$ & 43 \\
\hline Being asked added stress & $11 / 29$ & 38 & $17 / 42$ & 40 \\
\hline Felt pressure to consent & $9 / 29$ & 31 & $5 / 43$ & 12 \\
\hline \multicolumn{5}{|l|}{ Consent forms } \\
\hline Consent forms are necessary & $25 / 29$ & 86 & $35 / 42$ & 83 \\
\hline They are necessary to protect newborns & $24 / 29$ & 83 & $37 / 42$ & 88 \\
\hline Read consent form before deciding & $24 / 29$ & 83 & $42 / 44$ & 95 \\
\hline Given a copy of consent form & $19 / 29$ & 66 & $36 / 43$ & 84 \\
\hline Consent form too long & & & $0 / 44$ & 0 \\
\hline
\end{tabular}

newborns to be in the clinical trial; five completed survey forms, but the other nine refused both the clinical trial and the consent study. Overall $88 \%$ of the parents who consented to the clinical trial also consented to the consent study, whereas only $36 \%$ of those who refused the clinical trial consented to the consent survey. As such a small percentage of the non-consenting parents completed the consent survey, it is unlikely that their responses appropriately represent the group, and therefore their responses are not presented.

The ages of the mothers ranged from 14 to 40 years (mean 29.5 years); fathers had a mean age of 33.4 years. In 21 cases $(48 \%)$ the mother made the decision about consent for the clinical trial, and in 10 cases $(23 \%)$ it was the father; in 11 $(25 \%)$ it was both (no response was given in two). Twenty four mothers (55\%) were primiparous; 20 (45\%) were multiparous with between one and five previous children. All but four parents $(40 / 44,91 \%)$ had a high school diploma, and $34(77 \%)$ had post-secondary education. Five $(11 \%)$ had been in a research study previously, and seven (16\%) noted that someone in their family had been in a research study previously. Only two (5\%) respondents worked in health care. Twenty nine $(66 \%)$ had been admitted prenatally to the prenatal ward of Foothills Hospital, and only one newborn was transferred to the NICU from another hospital. This group of parents was similar to the parents surveyed in the retrospective study.

Tables 1 and 2 gives the results of the prospective study.

\section{Autonomy}

Most parents (91\%) were against the idea of the doctor making the decision to enrol the newborn into a research study and then informing the parents that their child was in a research study. Almost three quarters (70\%) of the parents did not feel pressure to enrol their newborn into the arginine trial.

\section{Level of comfort}

Most parents felt that they had made an informed decision and they were comfortable with the decision made. A significantly larger percentage of parents in this study (93\% v 59\%) said they were calm at the time that they made the decision to enrol their newborn into the arginine trial.

\section{Understanding around decision making}

All of the parents responded that they understood that they were giving consent for research as opposed to clinical care. As in the retrospective survey, most parents reported that they knew the purpose of the research and what procedures were involved in the study. Again, fewer parents (53\%) knew what alternatives were available or reported that they understood that there were risks associated with the research. Even though it was clearly stated in the consent form, only $90 \%$ of parents knew that they could withdraw the baby from the study. A significant percentage of the parents $(66 \%)$ felt that the baby would benefit directly from being in the study.

\section{Perception of the recruitment process}

(a) Timing: most parents had a chance to discuss enrolment of the newborn with their companion/spouse. A small number $(9 \%)$ felt that they were expected to give an immediate decision. More of the parents in the prospective study $(86 \% v$ $62 \%)$ reported that they had adequate time to ask all their questions.

(b) Consent forms: the views of the parents in the prospective study were not significantly different from those in the retrospective survey. Most responded that detailed consent forms were necessary $(83 \%)$, specifically for parents to protect their children $(88 \%)$ and they read the entire consent form before consenting (95\%) to the arginine trial. Only $84 \%$ remembered being given a copy of the consent form for their records, even though it is routine practice.

(c) Coercion: $95 \%$ of parents responded that they knew that they had a right to refuse to enrol their baby in the arginine trial. Less than half of the parents $(43 \%)$ replied that they had enrolled the baby into the study because they thought that the baby would get better care. Although $40 \%$ felt that being asked about enrolment into the research study increased their stress, only five parents ( $12 \%$ ) felt pressured to consent for their baby to be enrolled in the arginine trial.

\section{Parent-doctor communication}

Almost all (98\%) parents said that they felt comfortable to ask questions of the doctor, and that their questions were answered; most reported that the doctor used language that they could understand.

\section{Comparison of responses from retrospective survey and prospective study}

Tables 1 and 2 present summaries of the responses from the two studies. There is general agreement in responses from the two groups of parents. However, there are some significant differences between the two studies, which are probably related to the clinical trials for which the parents were being approached for potential enrolment of their newborns.

Under the theme of "level of comfort", there was a significant difference in the number of parents who reported being 
calm at the time that they made their decision to enrol their newborn into a clinical trial $(17 / 29 v 41 / 44, \mathrm{p}<0.001)$. In the prospective study, parents were approached when the newborn was 2 or 3 days old and had a day to make the decision about enrolment, whereas in the retrospective study there were a number of clinical trials for which the newborns were being recruited including a surfactant trial and a ventilator trial, both of which required decisions to be made more quickly.

Similarly, under the theme of "timing", there was a significant difference between the two studies, with more parents in the retrospective survey feeling that they had to make an immediate decision $(45 \% v 9 \%, \mathrm{p}<0.001)$. Fewer parents in the retrospective survey felt that there was adequate time for questions compared with the prospective study (62\% v 86\%, $\mathrm{p}=0.024)$. Under the category of "coercion", there was a tendency towards fewer parents reporting that they knew that they had a right to refuse participation in the clinical trial (79\% $v 95 \%, \mathrm{p}=0.054)$, and more parents feeling pressure to consent $(31 \% \vee 12 \%, \mathrm{p}=0.067)$. These tendencies may be related to the different clinical trials for which newborns were being recruited, differences in the acuity of the medical conditions being treated, and the limited alternatives available for treatment of the newborns.

\section{DISCUSSION}

This report includes the results of a retrospective survey and a prospective study of parents who enrolled their ill newborns into research studies in an NICU. The results of the two are very similar. Our respondents strongly believed that the parents should be the ones who decide about enrolment into research, not the doctors. For most parents, the request for consent for research did not "add stress to an already stressful situation", nor did most feel pressure to consent. This is contrary to what has been assumed by trialists, but consistent with other surveys of parents. ${ }^{72}$ A survey of European parents ${ }^{7}$ and a survey of Canadian parents ${ }^{12}$ of newborns support the value of consent for research, and that it is the parents' responsibility to decide.

Clinical trialists have argued that parents are not sufficiently educated to understand medical issues surrounding the research. ${ }^{3}$ The parents in this survey were reasonably well educated, with 91-93\% having graduated from high school ( 12 years of primary public schooling), and $59-77 \%$ having post-secondary education. Education level and age of mothers delivering low birthweight babies in our centre are higher than the Canadian national average. ${ }^{13}$ The need for informed consent has been suggested to act as a "social filter" biasing recruitment to the less well educated families. This does not appear to be the case for our population of parents. Furthermore, parents with less education may not have access to good health care for their children and therefore may attempt to access health care through participation in research. In Canada, health care is provided free of direct charge to all. Parents in our studies would not have felt pressured to enrol their newborns into trials in order to access health care. Therefore such arguments do not appear to be valid in Canada. Our results are similar to those of the other Canadian survey of parental decision making, which also found that sociodemographic characteristics did not play an important role. ${ }^{13}$ Analysis of their data suggested that perceptions of risk and benefit, and the integrity of the consent process were the most important factors influencing parents.

Critics have stated that parents may not be able to understand information given to them when their newborns are recruited to trials in the NICU. ${ }^{35}$ Although we did not objectively assess understanding in this study, the responses indicate that the parents understood, to their satisfaction, the purpose of the research and the procedures involved. However, fewer parents reported that they understood the alternatives to enrolment (53-76\%), the presence of risks (52-67\%), and that they could withdraw the baby from research (69-90\%). Therefore, the parents responding to this survey appeared satisfied with their understanding of the health and research issues surrounding the care and enrolment of their newborn(s) into research studies in the NICU. However, it may be that the explanations given to them about certain aspects of the research were inadequate for them to appropriately understand certain issues. This problem was clearly demonstrated in a study reported by Snowdon et al ${ }^{14}$ of parents who had enrolled their newborns into a randomised trial of extracorporeal membrane oxygenation versus conventional treatment. Interviews conducted when the infants averaged 97 weeks showed that the parents had serious gaps in their understanding of the concept of "randomisation".

Many alternatives to parental permission/consent for enrolment have been suggested as a means to increase enrolment in clinical trials in the NICU. Doctors or a designated administrative committee could act as surrogates and make the decision to enrol the newborn into a research trial and then inform the parents. ${ }^{46}$ An alternative form of recruitment is the "Zelen" method, in which patients are randomised and then consent is only requested from those who are randomised to the investigative arm of the trial. ${ }^{315}$ Supporters of parental consent have suggested that parents may become distressed and angry if this were instituted. Our results also do not support this type of presumptive enrolment. In a survey of parents specifically about Zelen randomisation, concerns were raised about the withholding of information from parents, the effect on decision making, the use of health information without parental knowledge or consent, and the long term impact on the parents and their ability to cope, particularly if the infant died. ${ }^{16}$

Our previous research into parental preferences on enrolment and consent showed that parents of either healthy or ill newborns did not want their newborn enrolled in even low risk studies without their consent. ${ }^{17}$ However, it has been reported that infants randomised to the placebo arm of a randomised controlled trial had better outcomes than infants who were eligible but were not enrolled into the study. ${ }^{18}$ Therefore it does appear that there may be a placebo effect even in this patient population, and that enroling the newborn into a trial may be in their best interests provided that the control arm provides the current best treatment. ${ }^{19}$

Parents in our studies suggested ways in which the process could be improved, including provision of information about appropriate trials before delivery so that there would be more time for reflection. They also suggested that researchers should provide clearer explanations of the background information supporting the reason for the trial, and that a member of the research team return the day after recruitment to review the information with the parent(s) or answer questions that may have subsequently arisen. The parents of twins have suggested that both twins be randomised together so that they both receive the same treatment. Owing to the large number of trials being conducted in the NICU, parents are often approached to enrol their newborn(s) into more than one study. The respondents were not against this, but most suggested that the number of trials in which it was appropriate for a newborn to be enrolled was two.

The retrospective survey was limited by the dependence on the parents' recollection of an event that may have occurred up to a year previously. They had been approached for one of several trials that were being conducted in the NICU and there would not have been enough responses for each of the trials to analyse them separately. Each clinical trial or study would have a different balance of risks and benefits as well as a different time requirement for reaching agreement for enroling the newborn. However, other studies into consent for studies in the NICU have been conducted several weeks to months after enrolment; our results are consistent with theirs. 
However, a prospective study asking parents about their understanding of the protocols and their concerns about enroling their newborns into trials shortly after the event would eliminate the loss of information from selective memory. Therefore a prospective study was conducted so that parents would have been exposed to the same information about the same trial and their newborns exposed to the same potential risks and benefits of a single clinical trial. Also, parents were approached 24 hours after enrolment so that the encounter was fresh in the memory.

The forms developed to survey parents in these studies could also be used for quality control assessments with the intent of gathering feedback from all parents who may have varied impressions of the recruitment and consent process. Over time this may provide feedback from a larger number of parents who might have different opinions on the recruitment process. This would be useful if different doctors or residents were involved in recruiting parents and newborns.

In conclusion, the parents who were surveyed in these studies were educated, understood the requests being made of them, and felt that they were able to focus on the issues and make informed decisions. These parents consented for their newborns to be enrolled into research studies, did not disapprove of the process, and strongly believed that the decision about enrolment should be theirs, not solely the doctors'. Although alternative methods of recruitment that exclude active participation of the parents have been suggested, we believe that it would be better to provide parents with the appropriate psychological support and information so as to maintain their competence as decision makers and assure them that their decision to consent, or refuse, to enrol their newborn(s) into a study would not negatively affect the care of their newborn(s). It would appear that we need to improve the consent process so that parents have a better understanding of the research risks and alternatives to participation in research.

\section{ACKNOWLEDGEMENTS}

This study was funded from the Ruth Rannie Memorial Fund, Faculty of Medicine.

\section{Authors' affiliations}

E Burgess, Office of Medical Bioethics, Faculty of Medicine, University of Calgary, Calgary, AB, Canada

N Singhal, H Amin, D D McMillan, Division of Neonatology, Faculty of Medicine, University of Calgary

H Devrome, Faculty of Law, University of Calgary

\section{REFERENCES}

1 NIH. Policy and guidelines on the inclusion of children as participants in research involving human subjects. Bethesda: $\mathrm{NIH}$, release date 6 March 1998

2 Silverman WA. The future of clinical experimentation in neonatal medicine. Pediatrics 1994:94:932-8.

3 Mason S. Obtaining informed consent for neonatal randomized controlled trials: an "elaborate ritual"? Arch Dis Child Fetal Neonatal Ed 1997;76:F143-5

4 Modi N. Clinical trials and neonatal intensive care.Arch Dis Child Fetal Neonatal Ed 1994:70:F231-2.

5 Editor. Your baby is in a trial. Lancet $1995: 345: 805-6$.

6 Rogers CG, Tyson JE, Kennedy KA, et al. Conventional consent with opting in versus simplified consent with opting out: an exploratory trial for studies that do not increase patient risk. J Pediatr 1998;132:606-1 1.

7 Mason SA, Allmark PJ, for the Euricon Study Group. Obtaining informed consent to neonatal randomized controlled trials: interviews with parents and clinicians in the Euricon study. Lancet 2000;356:2045-51.

8 Thong YH, Hart SC. The social filter effect of informed consent in clinical research. Pediatrics 1991;87:568-9.

9 Baum M. New approach for recruitment into randomized controlled trials. Lancet 1993;341:812-13.

10 Harth SC, Thong YH. Parental perceptions and attitudes about informed consent in clinical research involving children. Soc Sci Med 1995;40:1573-7.

11 Langley JM, Halperin SA, Mills EL, et al. Parental willingness to enter a child in a controlled vaccine trial. Clin Invest Med 1998;21:12-16.
12 Zupancic JAF, Gillie P, Streiner DL, et al. Determinants of parental authorization for involvement of newborn infants in clinical trials. Pediatrics 1997;99:e6.

13 Tough SC, Svenson LW, Johnston DW, et al. Characteristics of preterm delivery and low birthweight among 113994 infants in Alberta, 1994-1996. Can J Public Health 2001:92:276-80.

14 Snowdon C, Garcia J, Elbourne D. Making sense of randomization: responses of parents of critically ill babies to random allocation of treatment in a clinical trial. Soc Sci Med 1997;45:1337-55.

15 Zelen M. A new design for randomized clinical trials. N Engl J Med 1979:300:1242-5.

16 Snowdon C, Elbourne D, Garcia J. Zelen randomization: attitudes of parents participating in a neonatal clinical trial. Control Clin Trials 1999;20: 149-71.

17 Singhal N, Oberle K, Burgess E, et al. Parents' perceptions of research with newborns. J Perinatol 2002;22:57-63.

18 Schmidt B, Gillie P, Caco C, et al. Do sick newborn infants benefit from participation in a randomized clinical trial? J Pediatr 1999;134:151-5.

19 Allmark P, Mason S, Gill AB, et al. Is it in a neonate's best interest to enter a randomized controlled trial? J Med Ethics 2001;27:110-13.

\section{COMMENTARY}

The public, medical colleges, and the General Medical Council expect medicine to be practised to a high standard and where possible be evidence based. For this to occur, high quality research must necessarily be undertaken. Although some questions may be addressed by in vitro or animal studies, others can only be satisfactorily answered with research involving actual patients. Pivotal to the whole research process in patients is the issue of appropriate informed consent. ${ }^{1}$ This may be difficult enough when enrolling consent competent adults, but becomes even more difficult when approaching parents about enrolling their children. ${ }^{2}$ The research process is seen very differently by participating patients (or their parents) from the research team. ${ }^{3}$ Despite this, the views of study participants (and their families) have largely been ignored.

A poorly thought out approach to giving appropriate information about a study and obtaining consent is likely to result in patients either refusing to be enrolled or, perhaps worse still, enrolling without proper understanding of what is involved. The latter may result in events such as those seen recently. ${ }^{4}$ Failure to ask participants about the experience of being approached to take part in research misses important opportunities to improve research practice in the future. ${ }^{5}$

This paper by Burgess et al raises important issues about the consent process for research in the neonatal setting. This often involves approaching parents for consent at times of great stress, raising concerns over the validity of any consent obtained. There are suggestions that consent under these circumstances may be more valid if obtained from an independent source. Burgess et al used a combination of retrospective and prospective questionnaires to obtain information about parents' perspectives of the consent process. Although their methodology is not ideal because it involved several studies with differently timed interventions with different time intervals from the study to administration of the questionnaire, it highlights areas for future exploration.

It is clear that most parents wished to retain the right to give consent on behalf of their infants, even though this often adds to an already stressful situation. Other researchers have found similar views expressed by parents. ${ }^{6}$ Most responders felt that they had received adequate information about the studies, although prenatal information from and post-enrolment discussion with the research team would have been welcomed. Importantly, most parents appeared happy to be approached about enrolment in more than one study. This is often deemed inappropriate by ethics committees. No information is given about the very few questionnaire respondents who chose not to participate in the research studies.

These findings clearly need further study. Research undertaken in the neonatal setting ranges from potential life saving interventions offered as part of randomised controlled trials 
through observational follow up studies. Some interventions may be potentially non-therapeutic and involve uncomfortable procedures such as venepuncture. ${ }^{7}$ The issue of using healthy control infants may appear ethically difficult. It is essential that we obtain parents' views over this entire range of studies. Currently there is no obligation for researchers to assess the research experience of their subjects, although this has been proposed. ${ }^{5}$ One might liken this to "closing the loop" in the audit cycle. Future studies might easily incorporate standardised questionnaires for participants. Regrettably, the most difficult information to obtain concerns those parents who choose not to participate in research. Standardised questionnaires may shed some light on this important (often overlooked) group.

A C Fenton

Newcastle Neonatal Service, Ward 35, Royal Victoria Infirmary, Queen Victoria Road, Newcastle upon Tyne NE 1 4LP, UK; a.c.fenton@ncl.ac.uk

\section{REFERENCES}

1 General Medical Council. Seeking patients' consent: the ethical considerations. London: General Medical Council, 1998.

2 Royal College of Paediatrics and Child Health: Ethics Advisory Committee. Guidelines for the ethical conduct of medical research involving children. Arch Dis Child 2000;82:177-82.

3 Snowdon C, Garcia J, Elbourne D. Reactions of participants to the results of a randomised controlled trial: exploratory study. BM 1998;317:21-6.

4 NHS Executive West Midlands Regional Office. Report of a review of the research framework in North Staffordshire Hospital NHS Trust (Griffiths report). Leeds: NHS Executive, 2000.

5 Oesterheld J, Fogas B, Rutten S. Ethical standards for research on children. J Am Acad Child Adolesc Psychiatry 1998;37:684-5.

6 Snowdon C, Elbourne D, Garcia J. Zelen randomization: attitudes of parents participating in a neonatal clinical trial. Control Clin Trials 1999;20:149-71.

7 Shah VS, Al-Kharnan M, Quinn MW, et al. Is venepuncture in neonatal research ethical? Arch Dis Child 1997;77:F141-2.

\section{$\mathrm{BMJ}$

We are delighted to announce this forthcoming conference in Auckland, New Zealand.

The themes of the 3rd Asia Pacific Forum on Quality Improvement in Health Care are:

- Agenda for quality: Improving equity in health care delivery

- Improving safety

- Leadership for improvement

- Measuring quality and benchmarking for change

- Evidence based knowledge and education for quality improvement

- Improving health systems

- Patient/consumer centred quality improvement

Presented to you by the BM Publishing Group (London, UK) and Institute for Healthcare Improvement (Boston, USA), supported by the New Zealand Ministry of Health, ACC, and Standards New Zealand.

For more information about the Forum or to register contact: quality@bma.org.uk or go to:

www.quality.bmipg.com

Tel: +44 (0)2073836409 Fax: +44 (0)207383 6869 